\title{
Solution of Two Dimensional Poisson Equation Using Finite Difference Method with Uniform and Non-uniform Mesh Size
}

\author{
Genet Mekonnen Assefa (Msc) Lemi Guta (PhD) \\ Adama Science and Technology University Office of Graduate Studies
}

\begin{abstract}
This study focus on the finite difference approximation of two dimensional Poisson equation with uniform and non-uniform mesh size. The Poisson equation with uniform and non-uniform mesh size is a very powerful tool for modeling the behavior of electro-static systems, but unfortunately may not be solved analytically for very simplified models. Consequently, numerical simulation must be utilized in order to model the behavior of complex geometries with practical value. In most engineering problems are also coming from steady reactiondiffusion and heat transfer equation, in elasticity, fluid mechanics, electrostatics etc. the solution of meshing grid is non-uniform and uniform where fine grid is identified at the sensitive area of the simulation and coarse grid at the normal area.The discretization of non-uniform grid is done using Taylor expansion series. The purpose of such discretization is to transform the calculus problem to numerical form (as discrete equation). Therefore, in this study the two dimensional Poisson equation is discretazi with uniform and non-uniform mesh size using finite difference method for the comparison purpose. More over we also examine the ways that the two dimensional Poisson equation can be approximated by finite difference over non-uniform meshes, As result we obtain that for uniformly distributed gird point the finite difference method is very simple and sufficiently stable and converge to the exact solution whereas in non-uniformly distributed grid point the finite difference method is less stable, convergent and time consuming than the uniformly distributed grid points.
\end{abstract}

Keywords: Finite difference method, two dimensional Poisson equations, Uniform mesh size, Non-uniform mesh size, Convergence, Stability, Consistence.

DOI: $10.7176 /$ APTA/79-01

Publication date:September $30^{\text {th }} 2019$

\section{Introduction}

Numerous and diverse physical circumstances, and other mathematical problems are modeled in terms of partial differential equations (PDEs). As evident in the related literature PDEs of the Poisson type are among the most practical and frequently investigated. Therefore, in these study numerical solutions of linear 2-D Poisson equation is considered. Poisson equation is a generalization of Laplace's equation. The equation is named after the French mathematician geometer, and physicist Simon Denis Poisson. It is also a very powerful tool for modeling the behavior of electrostatic systems, but unfortunately may not only be solved analytically for very simplified models. Consequently, numerical simulation must be utilized in order to model the behavior of complex geometries with practical value. Although there are several competing algorithms for achieving this goal, one of the simplest and more straightforward of these is called the finite difference method (FDM).

Finite difference method is used as direct conversion of the partial differential equation from continuous function and operator into their discretely-sampled counterpart. This converts the entire problem into a system of linear equations that may be readily solved by means of matrix inversion, Jacobi, Gauss-elimination, successive over-relaxation method. The accuracy of such a method is therefore directly tied to the ability of a finite grid to approximate a continuous system and errors may be arbitrarily reduced by simply increasing the number of samples. A recent study (Gueye, S.B. 2014), Concerning the case of one dimension has proposed a direct, exact, and closed formulation of the inverse matrix. This inverse matrix has allowed getting a new, extremely fast solution to the Poisson equation. However, this innovative solution, obtained with the finite difference method, discussed only the case of boundary condition of type: Dirichlet boundary condition.

Regarding this partial differential equation we note that there are two main ways of finite difference discretization i.e. over a uniform grid where the mesh point is constant and over a non-uniform grid where the distance of the mesh point is not constant (Heraklion, February 2009). When we apply finite difference method to the continuous two-dimensional Poisson equation the equation is replaced by a "discrete" approximation. The number of those discrete points can be selected uniformly or non-uniformly depending on the mesh size $(h)$. The mesh is the set of locations where the discrete solution is computed. Two key parameters of the mesh are $\Delta x$ and $\Delta y$ the local distance between adjacent points in space. Finite difference discretization using equal mesh size in both $x$ and $y$ directions is simple to implement than non-uniform mesh size.In fact, non-uniform grids may give rise toa number of consistency/ stability phenomena that have no counterpart on uniform grids.Non-uniform meshes are often used for approximation of problems with generalized solution (B.Garcia-Archilla and J.M.Sanz-Serna 1991). In this case, the order of local erroris usually reduced. 


\subsection{Statement of the problem}

In (Karthik 2011 and James R.N 2012) the Poisson equation is solved using finite difference (FDM) with uniform mesh size in which the convergence, stability and the consistence are determined using the iterative method like Jacobi, Gauss-elimination, successive over-relaxation method etc. However, in particular case solving Poisson equation (2-D) with non-uniform mesh size is not considered in previous studies. And also the finite difference solution of two dimensional Poisson equation at the uniform and non-uniform mesh size are not compared. Therefore, this study will focus on finite difference solution of the two dimensional Poisson equation with both uniform and non-uniform mesh size for comparison purpose. Particularly, Dirichlet boundary conditions will be considered using the Finite difference method. Essentially, attention is given to the matrices extracted from the algebraic equations.

This study is intended to answer the following basic questions:

$>$ How to disicretaiz the two dimensional Poisson equation using finite difference method with nonuniform mesh size?

$>$ What will be the convergence, stability and consistence of finite difference method (FDM) with nonuniform mesh size?

$>$ How does the stability and consistency of FDM will be affected if the mesh size is non-uniform?

\subsection{Objective of the study}

\subsubsection{General Objective}

The general objective of this study is to obtain the finite difference solution of two dimensional Poisson equations with uniform and non-uniform mesh size and comparing each other.

\subsubsection{Specific Objectives}

The principal objectives of this study are:

$>$ To determine the convergence, stability and consistence of finite difference method for two dimensional Poisson equations with uniform and non-uniform mesh size.

$>$ To determine the effect of the mesh size on the convergence, stability and consistence of the finite difference method for two dimensional Poisson equation.

$>$ To identify the speed of convergence, stability and consistency of finite differencemethod with nonuniform mesh size discretization.

\subsection{Significance and Beneficiaries}

The finite difference solution of 2-D Poisson equation has great interest for a wide range of fields such as engineering, physics, mathematics, biology, chemistry, etc. And also it has broad applications in mechanical engineering, theoretical physics and other fields. Thus, solving and seeking an alternative method how to solve this equation will greatly benefit the scientific and industrial community.

\section{Literature Review}

In fluid dynamics, thermodynamics, solid mechanics etc. a large number of differential equations are found. And to solve them analytically is very difficult and at time it is impossible. Therefore, Finite difference method is used to solve the numerically to such equation. The value obtained from this method can be used in simulation of the problem. [G. Biswas,IIT Kanpur 2009].

Basically, 2-D Poisson equations are solved numerically by discretizing the problems to linear system. In order to obtain numerical solutions, some valid numerical methods for discretizing the problem of 2-D Poisson equation have been developed in recent years, including the mesh-free [Fletcher C.A.J, et.al. 1991] and meshbased methods.

The numerical techniques for the solution of two dimensional Elliptic partial differential equations such as Laplace's and Poisson's equations with uniform mesh size. These types of differential equations have specific applications in physical and engineering models. The discrete approximation of both equations is based on finite difference method. In this research, five points finite difference approximation is used for Laplace's and Poisson's equations. To solve the resulting finite difference approximation basic iterative methods; Jacobi, Gauss-Seidel and Successive over Relaxation (SOR) have been used. (Yousif Ahmed Qahraman July 2014)

Discretizing of the Poisson equation using any of the schemes that means the schemes that employ nonuniform grid and the scheme that employ uniform grids mostly leads to a sparse matrix. (Shukla and Zhong, 2005).

In most engineering problems, the solution of meshing grid is non-uniform where fine grid is identified at the sensitive area of the simulation and coarse grid at the normal area. The purpose of the experiment is to ensure the simulation is accurate and utilizes appropriate resources. The discretization of non-uniform grid was done using Taylor expansion series and Finite Difference Method (FDM). Central difference method was used to minimize the error on the effect of truncation. The purpose of discretization is to transform the calculus problem 
(as continuous equation) to numerical form (as discrete equation). [Andrew P. Wandel1. et.al. 2013]

\section{Methodology of the study}

\subsection{Instrumentation}

To do this thesis some instruments are needed like computer, and mat lab software.

\subsection{Study design and Procedures}

This section consists of methods and materials used to undertake the study. These are instrumentation and procedure of the study.

To attain the objective of the study, the following procedures are undertaken:

$>$ Generating the grids uniformly and non-uniformly/ defining the domain.

$>$ Replacing the given equation by the finite difference approximation.

$>$ Solving the tri-diagonal matrix that we get after discretiztion.

$>$ Validating the method using numerical examples.

$>$ Analyzes the solution.

$>$ Discussing the convergence, stability and consistence from the analysis.

\subsection{Finite difference discretization of two dimensional Poisson equation with uniform mesh size}

Now, finite difference method is used as direct conversion of the 2-D Poisson equation from continuous function and operator into their discretely-sampled counterpart. This converts the entire problem into a system of linear equations that may be readily solved by means of iterative method.

$$
\Delta^{2} U=-f(x, y)
$$

Where $\Delta$ stands for the Laplacian operator, and $\mathrm{f}$ is called as load/source function. The solution of the equation $\mathrm{U}$ is the unknown scalar potential function. In Cartesian coordinate system, the two dimensional Poisson equation is given by: $\frac{\partial^{2} u}{\partial x^{2}}+\frac{\partial^{2} u}{\partial y^{2}}=-f(x, y)$

Where $x, y, z$ are the independent space dimensions. There is no initial condition because the equation does not depend on time hence it becomes boundary value problem. Suppose that the domain is $D=(a, b) \times(c, d)$ and the domain is subject to Dirichlet boundary condition and it is covered by a square grid of size. Here there are some steps

Step 1: Generate a grid, in uniform Cartesian grid the grids can be generated as.

$x_{i}=a+i h_{x}, i=0,1,2,3, \ldots, M h_{x}=\frac{b-a}{M}$

$y_{j}=c+j h_{y}, \quad j=0,1,2,3, \ldots, M h_{y}=\frac{d-c}{M}$

We denote by $U$ a grid function whose value $U_{I J}$ at a typical point $\left(x_{i}, y_{j}\right)$ in domain $\mathrm{D}$ is intended to approximate the exact solution $U\left(x_{i}, y_{j}\right)=U(x, y)$ at that point. The solutionat the boundary node is known from the boundary condition (BCs) and the solution atthe internal grid point is to be approximated.

Step 2: Represent the partial derivative with FD formula involving the functional value at the grid point.

$$
\begin{gathered}
u_{x x}=\frac{u\left(x_{i+1}, y_{j}\right)-2 u\left(x_{i}, y_{j}\right)+u\left(x_{i-1}, y_{j}\right)}{\left(h_{x}\right)^{2}} \\
u_{y y}=\frac{u\left(x_{i}, y_{j+1}\right)-2 u\left(x_{i} y_{j}\right)+u\left(x_{i}, y_{j-1}\right)}{\left(h_{y}\right)^{2}}
\end{gathered}
$$

Therefore the 2-dimensional Poisson equation is approximated as bellow:$\frac{u\left(x_{i+1}, y_{j}\right)-2 u\left(x_{i}, y_{j}\right)+u\left(x_{i-1}, y_{j}\right)}{\left(h_{x}\right)^{2}}+\frac{u\left(x_{i}, y_{j+1}\right)-2 u\left(x_{i} y_{j}\right)+u\left(x_{i}, y_{j-1}\right)}{\left(h_{y}\right)^{2}}=-\left(f\left(x_{i}, y_{j}\right)+T_{i, j}\right)$ $i=1,2,3, \ldots M-1 \quad$ And $j=1,2,3, \ldots N-1$

The local truncation error satisfies.

$T_{i, j} \sim \frac{\left(h_{x}\right)^{2} \partial^{4} u}{12 \partial x^{4}}\left(x_{i}, y_{j}\right)+\frac{\left(h_{y}\right)^{2} \partial^{4} u}{12 \partial y^{4}}\left(x_{i}, y_{j}\right)+o\left(h^{4}\right)$

Where $h=\max \left(h_{x}, h_{y}\right)$

We ignore the error term and replace the exact solution values $u\left(x_{i}, y_{j}\right)$ at the grid points with the 
approximate solution values $u_{i_{i} j}$, that is

$$
\left(\frac{u_{i+1, j}+u_{i-1, j}}{\left(h_{x}\right)^{2}}+\frac{u_{i, j+1}+u_{i, j-1}}{\left(h_{y}\right)^{2}}\right)+\left(\frac{2}{\left(h_{x}\right)^{2}}+\frac{2}{\left(h_{y}\right)^{2}}\right) u_{i_{j} j}=-f\left(x_{i,}, y_{j}\right)
$$

The finite difference equation at the grid point $\left(x_{i}, y_{j}\right)$ involves five grid points in a five-point stencil $\left(x_{i+1}, y_{j}\right),\left(x_{i-1}, y_{j}\right),\left(x_{i}, y_{j-1}\right),\left(x_{i}, y_{j+1}\right)$, and $\left(x_{i}, y_{j}\right)$. the center $\left(x_{i}, y_{j}\right)$ is called the master grid point, where the finite difference equation is used to approximatethe PDE. Suppose that $h_{x}=h_{y}=$ $\mathrm{h}$, then equation (4) can be rearranged into.

$u_{i+1, j}+u_{i-1, j}-4 u_{i, j}+u_{i, j+1}+u_{i, j-1}=-h^{2} f\left(x_{i}, y_{j}\right)$

Step 3:Form the linear system of algebraic equations at a given gird points and solve by using iterative methods to get the approximate values for the solution at all grid points.

The matrix-vector form yields.

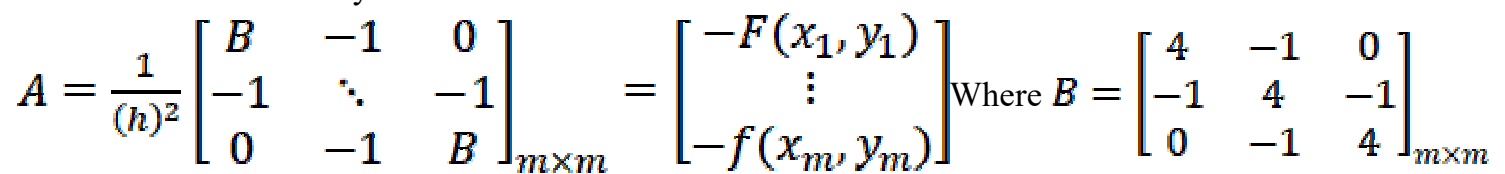

It is essential to store matrices A, B and an identity matrix I as "sparse" matrices onlythe non-zero entries are stored.

Step 4:Error analysis, implementation, visualization, etc.

Numerical example

Consider Poisson equation with uniform mesh size:-

$\frac{\partial^{2} \mathrm{u}}{\partial \mathrm{x}^{2}}+\frac{\partial^{2} \mathrm{u}}{\partial \mathrm{y}^{2}}=-2 \pi^{2} \sin (\pi \mathrm{x}) \sin (\pi \mathrm{y})$

For $0 \leq x \leq 1$ and $0 \leq y \leq 1$ with Dirichlet boundary condition on all sides of $\mathrm{f}$ unite square that is $u(0, y)=u(1,0)=u(x, 0)=u(x, 1)=0$ and let $h=k=\frac{1}{4}$

The exact solution is $u(x, y)=\sin (\pi x) \sin (\pi y)$

Therefore we have spares matrix A as flows:

$\left[\begin{array}{ccccccccc}4 & -1 & 0 & -1 & 0 & 0 & 0 & 0 & 0 \\ -1 & 4 & -1 & 0 & -1 & 0 & 0 & 0 & 0 \\ 0 & -1 & 4 & 0 & 0 & -1 & 0 & 0 & 0 \\ -1 & 0 & 0 & 4 & -1 & 0 & -1 & 0 & 0 \\ 0 & -1 & 0 & -1 & 4 & -1 & 0 & -1 & 0 \\ 0 & 0 & -1 & 0 & -1 & 4 & 0 & 0 & -1 \\ 0 & 0 & 0 & -1 & 0 & 0 & 4 & -1 & 0 \\ 0 & 0 & 0 & 0 & -1 & 0 & -1 & 4 & -1 \\ 0 & 0 & 0 & 0 & 0 & -1 & 0 & -1 & 4\end{array}\right]\left[\begin{array}{c}u_{1,1} \\ u_{2,1} \\ u_{3,1} \\ u_{1,2} \\ u_{2,2} \\ u_{3,2} \\ u_{1,3} \\ u_{2,3} \\ u_{3,3}\end{array}\right]=\left[\begin{array}{c}0: 61685 \\ 0: 872358 \\ 0: 61685 \\ 0: 872358 \\ 1: 2337 \\ 0: 87235 \\ 0: 61685 \\ 0: 872358 \\ 0: 61685\end{array}\right]$

We can use different iterative methods to solve this matrix but this study we use matrixinverse method by using mat-lab we get the following solution. 
Table 1: Comparison of analytical solution with the approximate solution/ finite difference solution with uniform mesh

\begin{tabular}{|l|l|l|l|}
\hline$(x, y)$ & Exact solution & Approximate solution & Error \\
\hline$\left(\frac{1}{4}, \frac{1}{4}\right)$ & 0.5 & 0.5265 & 0.0265 \\
\hline$\left(\frac{1}{2}, \frac{1}{4}\right)$ & 0.7071 & 0.7446 & 0.0375 \\
\hline$\left(\frac{3}{4}, \frac{1}{4}\right)$ & 0.5 & 0.5265 & 0.0265 \\
\hline$\left(\frac{1}{4}, \frac{1}{2}\right)$ & 0.7071 & 0.7446 & 0.0375 \\
\hline$\left(\frac{1}{2}, \frac{1}{2}\right)$ & 1 & 1.0530 & 0.0530 \\
\hline$\left(\frac{3}{4}, \frac{1}{2}\right)$ & 0.7071 & 0.7446 & 0.0375 \\
\hline$\left(\frac{1}{4}, \frac{3}{4}\right)$ & 0.5 & 0.5265 & 0.0265 \\
\hline$\left(\frac{1}{2}, \frac{3}{4}\right)$ & 0.7071 & 0.7446 & 0.0375 \\
\hline$\left(\frac{3}{4}, \frac{3}{4}\right)$ & 0.5 & 0.5265 & 0.0265 \\
\hline
\end{tabular}

As we observe from the table the solution is done for only the first iteration at $n=0$ the error we get is too small but to generalize the stability, consistence and the convergence of the method it is difficult so we can check for maximum number of iteration using mat-lab code.

\subsection{The finite difference discretization of two dimensional Poisson equations with non-uniform mesh size} The discretization of two dimensional Poisson equations with non-uniform grid is done by using Taylor expansion series and finite difference method. It may give rise to a number of consistency or stability phenomena that have no counterpart on uniform grid. This discretization is used to transform calculus problem to numerical form like uniform mesh. In this case there are two different ways of generating a grid, in the first method the non-uniform grid is generated at first and all the calculation are done by means of the operators given above and the second way, which is used in the code. Generally for small and simple fluid flow problem uniform grid can be used but turbulent combustion modeling which is very complex process, requiring the application of non-uniform gird. There are some steps to discretiz the two dimensional Poisson equation with non-uniform mesh like uniform mesh.

Step 1: Generate a grid. A non-uniform Cartesian grid can be generated as:-

$$
\begin{aligned}
& x_{i}=h_{x} \times \operatorname{rand}(1) \quad i=0,1,2,3, \ldots, M \quad h_{x i}=x_{i+1, j}-x_{i_{i} j} \text { in this case } j=0 \\
& y_{j}=k_{y} \times \operatorname{rand}(1) \quad j=0,1,2,3, \ldots, M \quad k_{y j}=y_{i, j+1}-y_{i, j} \text { in this case } i=0
\end{aligned}
$$

Step 2: At a typical internal grid point $u\left(x_{i}, y_{j}\right)$ we approximate the partial derivatives of u by second order central difference.

$$
u_{x x}+u_{y y}=-f(x, y)(1)
$$

So far, there is no difference between uniform and non-uniform meshes except for the subscript to the mesh spacing. Similarly evaluate the second derivative using the central difference scheme. Since we have:

$$
\begin{array}{r}
u_{x x}=\left(\frac{u_{i+1, j}-u_{i, j}}{h_{x i}}-\frac{u_{i, j}-u_{i-1, j}}{h_{x i-1}}\right)\left(\frac{2}{h_{x i}+h_{x i-1}}\right) \\
\text { And } u_{y y}=\left(\frac{u_{i, j+1}-u_{i, j}}{k_{y i}}-\frac{u_{i, j}-u_{i, j-1}}{k_{y j-1}}\right)\left(\frac{2}{k_{y j}+k_{y j-1}}\right)
\end{array}
$$

Results in the above central differences formula for the second derivative on a non-uniform mesh. Therefore the two dimensional Poisson equation can be discretaized as:- 


$$
\begin{aligned}
& u_{x x}+u_{y y}=\left(\frac{u_{i+1, j}-u_{i, j}}{h_{i}}-\frac{u_{i, j}-u_{i-1, j}}{h_{i-1}}\right)\left(\frac{2}{h_{i}+h_{i-1}}\right)+\left(\frac{u_{i, j+1}-u_{i, j}}{k_{j}}-\right. \\
& \left.\frac{u_{i, j}-u_{i, j-1}}{k_{i-1}}\right)\left(\frac{2}{k_{j}+k_{i-1}}\right)=-f_{i, j}
\end{aligned}
$$

(4)

Step 3: Form the linear system of algebraic equations at a given grid points and solve it by formulating a matrix to get the approximate values for the solution at all grid points.

The $9 \times 9$ matrix format looks like the following.

$$
\left[\begin{array}{ccc}
a_{1} & -a_{2} & 0 \\
-b_{1} & \ddots & -n_{4} \\
0 & -m_{2} & m_{3}
\end{array}\right]\left[\begin{array}{c}
u_{1,1} \\
\vdots \\
u_{3,3}
\end{array}\right]=h^{2}\left[\begin{array}{c}
f_{1,1} \\
\vdots \\
f_{3,3}
\end{array}\right]\left[\begin{array}{c}
u_{1,0}+u_{0,1} \\
\vdots \\
u_{3,4}+u_{4,3}
\end{array}\right]
$$

Where $a_{1} \neq 4, m_{3} \neq 4,-b_{1},-a_{2},-n_{4},-m_{2}, \ldots$ those all are different from - 1 because the value of the mesh size $h$ and $k$ are not constant and they are not equal.

Step 4: Error analysis, implementation, visualization, etc. In the above expression, the leading term in the truncation error is proportional to the difference between successive mesh spacing $\mathrm{s}$ and the scheme is therefore of first order accuracy if $\Delta x_{i} \sim 2 \Delta x_{i-1}$ Thus, there is a general loss of accuracy on non-uniform meshes if care is not exercised in deriving the approximations.

\section{Numerical Example}

Consider Poisson equation with non-uniform mesh size:-

$$
\begin{aligned}
& \frac{\partial^{2} \mathrm{u}}{\partial \mathrm{x}^{2}}+\frac{\partial^{2} \mathrm{u}}{\partial \mathrm{y}^{2}}=-2 \pi^{2} \sin (\pi \mathrm{x}) \sin (\pi \mathrm{y}) \\
& \text { For } 0 \leq x \leq 1 \text { and } 0 \leq y \leq 1 \\
& \begin{array}{lllll}
\mathrm{i} x_{i} y_{j} h_{i} k_{j} & & & \\
1 & 0 & 0 & 0.1270 & 0.0975 \\
2 & 0.1270 & 0.0975 & 0.5054 & 0.181 \\
3 & 0.6324 & 0.2785 & 0.1823 & 0.2684 \\
4 & 1 & 1 & &
\end{array}
\end{aligned}
$$

$\begin{array}{lllll}3 & 0.6324 & 0.2785 & 0.1823 & 0.2684\end{array}$

Where the mesh size $\mathrm{h}$ and $\mathrm{k}$ are randomly distributed in $x$ and $y$ direction respectively between 0 and 1 with Dirichlet boundary condition on all sides of $f$ unite square that is $u(0, y)=u(1,0)=u(x, 0)=u(x, 1)=0 \quad$. And the exact solution is $u(x, y)=\sin (\pi x) \sin (\pi y)$

Therefore we have spares matrix becomes as follows:-

$\left[\begin{array}{ccccccccc}0.029 & -0.004 & 0 & -0.008 & 0 & 0 & 0 & 0 & 0 \\ -0.002 & 0.031 & -0.005 & 0 & -0.013 & 0 & 0 & 0 & 0 \\ 0 & -0.001 & 0.005 & 0 & 0 & -0.001 & 0 & 0 & 0 \\ -0.022 & 0 & 0 & 0.064 & -0.006 & 0 & -0.015 & 0 & 0 \\ 0 & -0.036 & 0 & -0.008 & 0.087 & -0.0023 & 0 & -0.003 & 0 \\ 0 & 0 & -0.003 & 0 & -0.004 & 0.0161 & 0 & 0 & -0.001 \\ 0 & 0 & 0 & -0.034 & 0 & 0 & 0.1498 & -0.019 & 0 \\ 0 & 0 & 0 & 0 & -0.052 & 0 & -0.0269 & 0.189 & -0.076 \\ 0 & 0 & 0 & 0 & 0 & -0.0037 & 0 & -0.012 & 0.047\end{array}\right]\left[\begin{array}{c}u_{1,1} \\ u_{2,1} \\ u_{3,1} \\ u_{1,2} \\ u_{2,2} \\ u_{3,2} \\ u_{1,3} \\ u_{2,3} \\ u_{3,3}\end{array}\right]=\left[\begin{array}{c}0.005 \\ 0.002 \\ 0 \\ 0.005 \\ 0.019 \\ 0.001 \\ 0.023 \\ 0.085 \\ 0.04\end{array}\right]$

We can solve this matrix using different iterative methods or we can use spars matrix solver. For this study we use matrix inverse method by using mat-lab code. As we observe from the table the solution is done for only the first iteration at $\mathrm{n}=0$ 
Table 2: Comparison of analytical solution with the approximate solution/ finite difference solution with nonuniform mesh size

\begin{tabular}{|l|l|l|l|}
\hline$(x, y)$ & Exact solution & Approximate solution & Error \\
\hline$(0.1270,0.0975)$ & 0.1171 & 0.0820 & 0.0351 \\
\hline$(0.6324,0.0975)$ & 0.2758 & 0.2066 & 0.0692 \\
\hline$(0.8147,0.0975)$ & 0.1659 & 0.0644 & 0.1015 \\
\hline$(0.1270,0.2785)$ & 0.2982 & 0.2066 & 0.0916 \\
\hline$(0.6324,0.2785)$ & 0.7020 & 0.3488 & 0.3532 \\
\hline$(0.8147,0.2785)$ & 0.2924 & 0.1655 & 0.1269 \\
\hline$(0.1270,0.5469)$ & 0.3842 & 0.2883 & 0.0959 \\
\hline$(0.6324,0.5469)$ & 0.9048 & 0.6943 & 0.2105 \\
\hline$(0.8147,0.5469)$ & 0.5438 & 0.4705 & 0.0733 \\
\hline
\end{tabular}

To generalize the stability, consistence and the convergence of the method it is difficult like uniform mesh for non-uniform mesh we can check for maximum number of iteration using mat-lab code.

\section{Result and Discussion}

In this study we discuss the finite difference solution of two dimensional Poisson equation with uniform and non-uniform mesh. We analysis some of their property like consistency, stability, convergence and order of accuracy using mainly their modified/ discretized equation as our tool. We get the following four figures as result using MATLAB code for maximum number of iteration. Fig 4 is solution of the problem by finite difference method on non-uniform mesh whereas Fig 2 is solution the problem by finite difference method on uniform mesh and the other two figures Fig 1 and Fig 3 are exact solution for uniform mesh size and for nonuniform mesh size respectively.

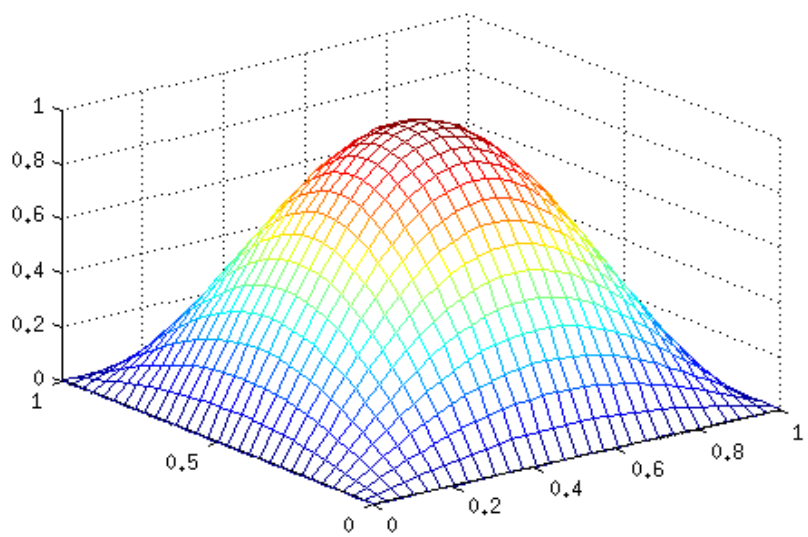

Figure 1 Exact solution on uniform mesh size

As we observe from the Fig 2 the finite difference solution of a given two dimensional Poisson equation is converge to the exact solution (Fig 1). From this we can also understand that the method is both consistence and stability depending on Lax-Richtmyer equivalence theorem. 


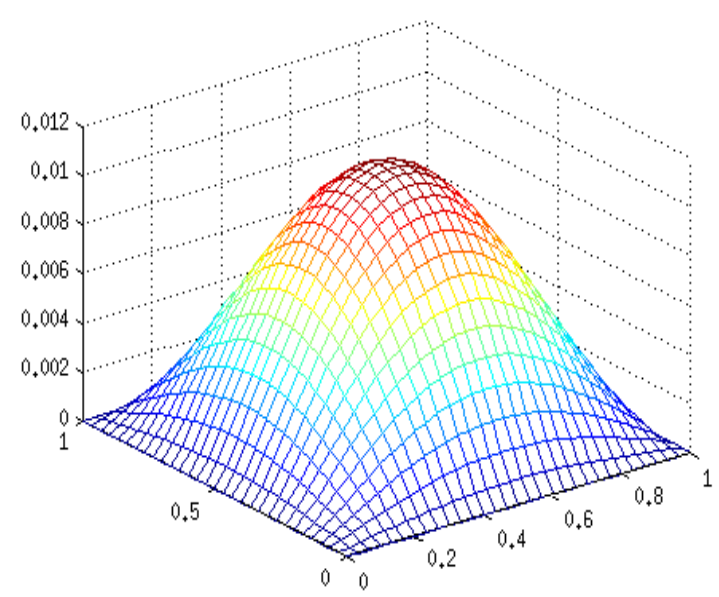

Figure 2 Approximate solutions on uniform mesh

Generally in this Fig 2 we understand that finite difference method for two dimensional Poisson equation with uniform mesh size is consistence, stable and convergent and also it is second order accurate. The smaller the step size gives more accurate numerical value. Trade-off between step size and computational effort is an issue in finite difference method. Therefore the step size definitely has strong effect on the accuracy of finite difference method of two dimensional Poisson equations.

As we observe from the Fig 4 below the finite difference solution on the non-uniform mesh is convergent but not exactly converge to the exact solution it vanish at some point because the data we distribute is not smoothly distributed and the mesh size is not decreasing that means it fluctuate from the first to the next grid point. Generally because of the data not distributed smoothly (equally) the Fig 4 shows the finite difference method on the given Poisson equation with non-uniform mesh is not exactly converge to the exact solution.

As a result in this experiment we have seen that the usual stability, consistency and convergence of finite difference scheme for two dimensional Poisson equation is not more sufficient when the mesh is non-uniform if the data are not smoothly distributed as we observe from Fig 4 But if we generate the data smoothly the method on non-uniform mesh is convergent. This shows that the mesh has a great effect on the stability, consistency and convergence of finite difference scheme. Comparison between non-uniform Vsuniform mesh case both have stability property of finite difference scheme for 2-D Poisson equation. We also note that in the uniform mesh case the order of accuracy of the finite difference approximation for 2D Poisson equation is higher than in

the finite difference approximation for 2-d Poisson equation with non-uniform mesh case. The reason for this accuracy decrease is the lack of symmetry of the non-uniform meshes. In the uniform mesh case the mesh does not change from the first step to the last step so there is no need for construction of new mesh. Where as in non-uniform mesh case the finite difference scheme become complicated significantly both the computation and the analysis of numerical approximation and theirproperties.

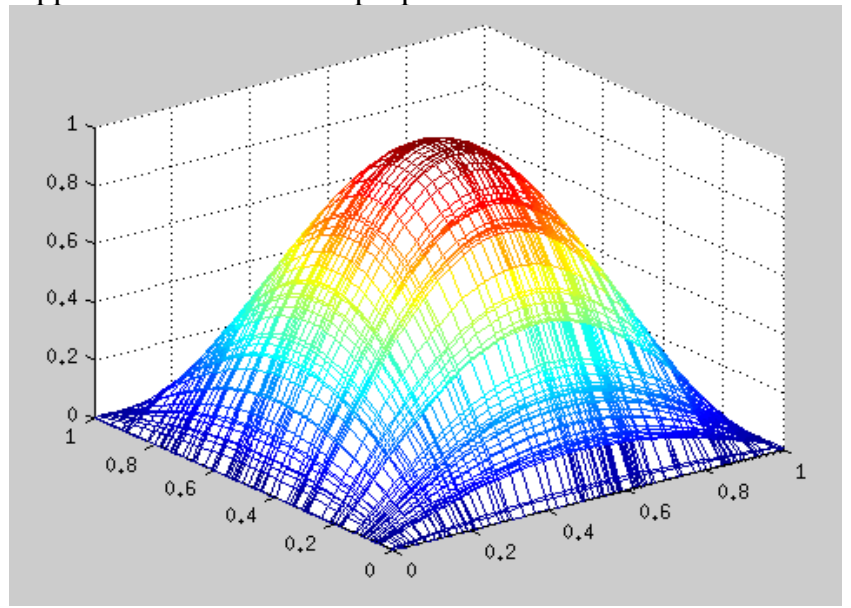

Figure 3 Exact solutions on non-uniform mesh size 


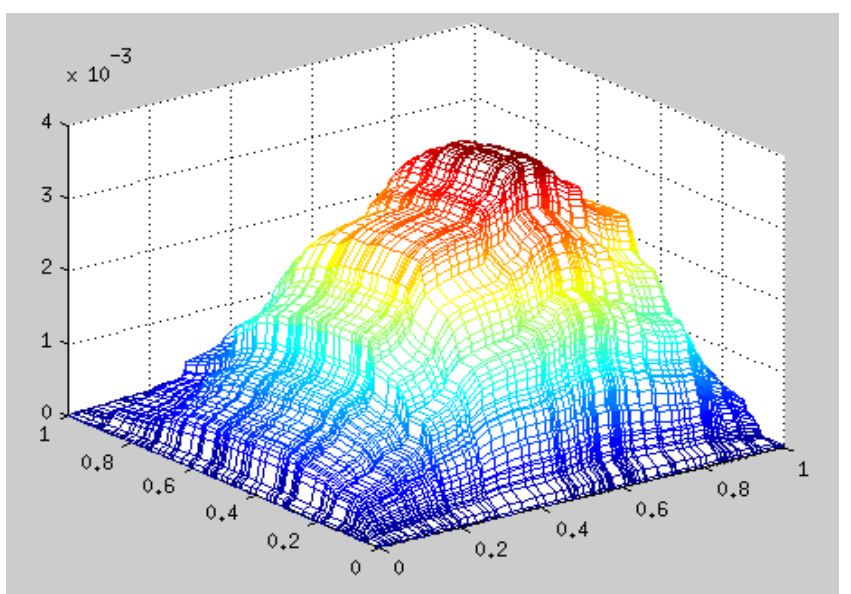

Figure 4 Approximate solutions on non-uniform mesh size

Usually the spitail derivatives of smooth function can be approximated over this mesh.The smaller the step size will give the more accurate numerical value. Trade-off between step size and computational effort is an issue in finite difference method. Generally for smoothly distributed the non-uniform mesh is more preferable than uniform mesh the reason is that the finite difference method for the two dimensional Poisson equation with non-uniform mesh is more convergent than with uniform mesh

\section{Conclusion and Recommendation \\ 5.1 Conclusion}

The 2-dimensional Poisson equation is discretized in order to transform the calculus partial differential equation to algebra discrete equation. The discretization process of 2- dimensional Poisson equation is by using Taylor expansion. For the small and simple fluid flow problem, uniform grid can be used, but turbulent combustion modeling, which is a very complex process, requiring the application of non-uniform grid meshing. The term by term discretization can be coded into MAT-LAB software.Generally in this thesis we understand the basic difference of uniform and non-uniform mesh size and their generation in two dimensions. From our result we know that for not smoothly distributed data point the convergence, stability and Constance of finite difference method for two dimensional Poisson equation with non-uniform mesh is not sufficiently accurate than finite difference method for 2-dimensional Poisson equation with uniform mesh size.

\subsection{Recommendation}

The result obtained in this study is on the solution of two dimensional Poisson equations using finite difference approximation with uniform and non-uniform mesh size for compering the result of uniform and non-uniform mesh.

This study can be extended further.

One may make analysis of this method for higher dimension with non-uniform mesh and can identify the stability, consistence and convergence and can make comparison with uniform mesh size.

\section{Bibliography}

$>$ Chew, C.S. (2006). A Generalized Finite-Difference (GFD) ALE scheme for in compressible flows around moving solid bodies on hybrid mesh free Cartesian grids. Journal of Computational Physics, 218: $510-545$.

D Evans D. J. (1997) Group Explicit Methods for the Numerical Solutions of Partial Differential Equations, Gordon and Breach Science Publishers, Australia.

> GayazKhakimzyanovG.K (2017) On supra convergence phenomenon for second order centered finite differences on non-uniform grids, Institute of Computational Technologies, Novosibirsk, Russia. Last modified: February 6, 2017.

$>$ Izadian, J. (2012) New Method for Solving Poisson Equation on Irregular Domains. App. Math. Science, 6: 369-380,

> Sukumar N. and Bolander J. E. (2003) Numerical Computation of Discrete Differential Operators on Non-Uniform Grids Department of Civil and Environmental Engineering, One Shields Avenue, University of California, Davis,U.S.A, CA 95616 ,

> Urena, K.F.(2011) Application of the generalized finite difference method to solve the advectiondiffusion equation, Journal of Computational Applied Mathematics 235.

> Karthik.V.(2011) 3-D ADI Poisson Solver Supercomputer Education and Research Center Indian 
Institute of Science BANGALORE 560012

Dang Evans W.E and Wang X.-P (2000), Numerical methods for the Landau-Lifshitz equation, SIAM J Sci Comp, 16471665.

$>$ Yousif W.S. and Evans D.J. (1995), Explicit Decoupled Group Iterative Methods and Their Implementations, Parallel Algorithms and Applications, 53-71.

> Yousif W.S. and Martins M.M. (2008), Explicit De-couple Group AOR method for solving elliptic partial differential equations, Neural, Parallel and Scientific Computations, no. 4, 531-542. 